\title{
Editorial
}

\section{Etiological Spectrum of Lower Gastrointestinal Bleeding: Geographical Location Does Matter}

\author{
Saroj K. Sinha ${ }^{1}$ Anupam K. Singh ${ }^{1}$ \\ ${ }^{1}$ Department of Gastroenterology, PGIMER, Chandigarh, India
}

J Digest Endosc 2019;10:163-165

Gastrointestinal (GI) bleeding is one of the most common medical and surgical emergencies in the gastroenterology practice. GI bleeding can occur from esophagus, stomach, or duodenum till ligament of Treitz, and is traditionally defined as upper gastrointestinal bleeding (UGIB). Bleeding from rest of the small bowel and colon is traditionally called lower gastrointestinal bleeding (LGIB). LGIB is approximately one-fifth as common as UGIB. ${ }^{1}$ The incidence and risk of lower GI bleeding increases with age, with a 200 -fold rise in incidence from the third decade to ninth decade of life. ${ }^{1}$ Multiple hypotheses have been put forward for this increase, presumably due to increasing incidence of diverticulosis, ischemic diseases involving bowel, and drug use in later part of life. ${ }^{2}$ Despite the increasing incidence of LGIB, the literature regarding the LGIB lags considerably behind UGIB.

Acute LGIB is arbitrarily defined as bleeding of fewer than 3 days in duration that may result in instability of vital signs, anemia, and/or need for blood transfusion. ${ }^{3}$ The definition of chronic LGIB is rather broad, encompassing passage of blood per rectum over a period of several days or longer implying intermittent or slow loss of blood. ${ }^{4}$ Patients with chronic bleeding can also have occult fecal blood loss presenting as anemia and is diagnosed as LGIB only when investigated.

LGIB may be arbitrarily grouped in several categories: anatomical (diverticulosis), inflammatory (inflammatory bowel disease, infectious colitis, etc.), vascular (ischemic, angiodysplasia, radiation induced), and neoplastic. While most of the studies continue to focus on UGIB, an increasing number of studies have addressed spectrum, etiology, and management of LGIB.

In this issue of the Journal of Digestive Endoscopy, Lakhanpal et al present the clinical profile of the chronic lower GI bleeding at sub-Himalayas. ${ }^{5}$ Out of 138 patients included in the study, $70.3 \%$ of the patients belonged to the age group of 40 to 59 years, which deviates from other studies where most of the patients belong to older age group. ${ }^{1,6}$ Hematochezia (97.8\%) was the most common presentation in this study, which is in agreement with the previously published work from the same region. ${ }^{6.7}$ Common etiologies identified in the study were hemorrhoids (19.5\%), inflammatory bowel disease (IBD) (19.5\%), colorectal carcinoma (17.4\%), infective causes (11.6\%), and radiation proctitis (9.4\%). In patients with age $<60$ years most common cause was IBD while in patients $>60$ years was carcinoma colon. Most of these etiologies are consistent with the other published paper from India, while few are showing rising trend. ${ }^{7}$ Compared with previous studies, colorectal carcinoma is showing increasing percentage among the LGIB etiologies. Dar et al in a retrospective study also found colorectal carcinoma (29.3\%) being the most common cause in North India. ${ }^{6}$

When comparing with other international studies for LGIB, the etiological diseases identified by Lakhanpal et al differ. Study from the western world by Longstreth et al showed the common causes of LGIB as diverticulosis (41.6\%), colorectal cancer (9.1\%), and ischemic colitis (8.7\%). ${ }^{1}$ Bai et al reviewed literature of 53,951 patients of LGIB and the most common etiology was colorectal cancer (24.4\%) followed by colorectal polyp (24.1\%), colitis (16.8\%), anorectal disease (9.8\%), and $\operatorname{IBD}(9.5 \%){ }^{8}$ Bai et al also found colorectal cancer being the most common cause of LGIB in both adults (24\%) and elderly patients (36\%). Different causes of LGIB in these studies suggest the difference in etiologies based on geographical variation in the prevalence of these conditions.

The work by Lakhanpal et al highlights the changing trend in etiological profile of LGIB in India and approaching similar to the western world. Etiology for LGIB varies according to the age group of the patients.

Among the varied causes for LGIB, diagnosis of LGIB starts with the symptom assessment. Bright red blood most commonly indicates a distal source (unless associated with hemodynamic instability), whereas black stool indicates a more proximal source. ${ }^{9}$ Painless bleeding is typically caused by diverticular bleeding, or vascular ectasia. However, abdominal pain is associated with inflammatory or ischemic cause of bleeding.
Address for correspondence Saroj K. Sinha, MD (Medicine), DM (Gastroenterology), Department of Gastroenterology, PGIMER, Chandigarh 160012, India (e-mail: sarojksinha@hotmail.com).
DOI https://doi.org/ 10.1055/s-0039-3401401 ISSN 0976-5042.
Copyright $\odot 2019$ Society of

Gastrointestinal Endoscopy of India
License terms

(요 (1) $\Theta \circledast$ 
In general, the investigational approach to LGIB is still not standardized. In approximately $15 \%$ of the cases, the source can be found in upper GI tract and esophagogastroduodenoscopy is the definitive test, when indicated. Some authors believe that routine anoscopy should be performed as a part of physical examination, as clue by the paper presented by Lakhanpal where $19.5 \%$ patients were having hemorrhoids as the cause of LGIB. ${ }^{2,5}$ This paper showed hematochezia as the commonest presentation and the commonest site involved being anorectum (19.5\%), whether flexible sigmoidoscopy be routinely used in place of anoscopy is yet to be answered. Sigmoidoscopy may be diagnostic for ulcerative colitis, infectious colitis, hemorrhoids, proctitis, or solitary rectal ulcer, obviating the need for colonoscopy in emergency.

Colonoscopy in vast majority of cases identifies the source of bleeding, provides the best opportunity for early diagnosis, and also for therapeutic intervention. Studies have shown the safety of early colonoscopy within 12 hours of admission in LGIB, and also help in identifying low risk patients and thus reduce the need for hospitalization and costs of care..$^{10}$ In the present study, colonoscopy was done in all the patients and $92.7 \%$ sources could be identified with colonoscopy only, highlighting the usefulness of colonoscopy in the management of lower GI bleeding.

Despite usefulness of colonoscopy, a subset of patients (3.5-15\%) still remains undiagnosed for source of LGIB and requires other investigation..$^{5-7}$ Other investigations include computed tomographic angiography, red blood cell scintigraphy, and digital subtraction angiography. ${ }^{11}$ Once diagnostic testing has been undertaken, specific therapy can be performed. For those undergoing colonoscopy and specific lesions with bleeding stigmata are identified, definite endoscopic therapies can be performed. Treatment of other disorders including hemorrhoids, various colitis, and malignancy depends on the specific abnormality.

Aspirin, clopidogrel, and similar antiplatelet drugs have become one of the most commonly used drugs over the last 1 or 2 decades. These are known to increase the risk of both upper and lower GI bleeding. ${ }^{12,13}$ Thus, this information becomes crucial when evaluating a patient with upper and lower GI bleed. Unfortunately, this data has not been clearly shown in the present study. ${ }^{5}$ Proton pump inhibitors are known the reduce the risk of initial and recurrent UGIB with these drugs. But there is no counterpart of proton pump inhibitors with similar efficacy in lower gastrointestinal tract. Further, the endoscopic hemostasis procedures are less commonly performed in lower gastrointestinal tract compared with upper gastrointestinal tract. Thereby, rules for stopping and restarting the antiplatelet drugs after an episode of lower GI bleeding remain less clearly defined. The current study does not provide any information about the events and outcome after the index admission or presentation. In one of the previous studies, readmission was required in $29 \%$ of the patients and the predictors for 30-day readmission for lower gastrointestinal bleed (LGIB) included metastatic disease, liver disease, and drug abuse. ${ }^{14}$ Most of the readmissions were not due to recurrent lower GI bleeding.
Angiography and transcatheter embolization are important modalities in the treatment of lower GI bleeding and it can be lifesaving in many situations, particularly when endoscopic intervention fails or is not feasible due to difficulties related to bowel preparation or hemodynamic instability. ${ }^{15} \mathrm{It}$ is not clear how many patients in the current study required angiography and apparently no patient underwent angioembolization. ${ }^{5}$ This remains unexplained but may also be due to selection bias. The current study also did not utilize any of the scoring system which has been described to predict the outcome of LGIB..$^{16,17}$

Etiological spectrum of lower GI bleeding depends on a lot of factors like age, comorbidities, use of drugs and medications, but geographical location is also an important factor which determines the spectrum. ${ }^{18-20}$ Despite the limitations inherent to the study, authors of the current study have presented a useful data which will be useful for the clinician of the concerned field.

\section{Conflict of Interest}

None declared.

\section{References}

1 Longstreth GF. Epidemiology and outcome of patients hospitalized with acute lower gastrointestinal hemorrhage: a population-based study. Am J Gastroenterol 1997;92(3):419-424

2 Rockey DC. Lower gastrointestinal bleeding. Gastroenterology 2006;130(1):165-171

3 Leerdam M, Ramsoekh D, Rauws E. Epidemiology of acute lower gastrointestinal bleeding. Gastrointest Endosc 2003;57:93

4 Jovanovic I, Milosavljevic T. Endoscopic therapy for lower gastrointestinal bleeding. Interventional and therapeutic gastrointestinal endoscopy. Front Gastrointest Res 2010;27:240-253

5 Lakhanpal V, Sharma R, Bodh V, Thakur S, Sharma N, Sharma B. Clinical spectrum of chronic lower gastrointestinal bleeding at sub Himalayas: a study at tertiary care hospital of north India. J Dig Endosc 2019, this issue

6 Dar IA, Dar WR, Khan MA, et al. Etiology, clinical presentation, diagnosis and management of lower gastrointestinal bleed in a tertiary care hospital in India: a retrospective study. J Dig Endosc. 2015;6:101-109

7 Goenka MK, Kochhar R, Mehta SK. Spectrum of lower gastrointestinal hemorrhage: an endoscopic study of 166 patients. Indian J Gastroenterol 1993;12(4):129-131

8 Bai Y, Peng J, Gao J, Zou DW, Li ZS. Epidemiology of lower gastrointestinal bleeding in China: single-center series and systematic analysis of Chinese literature with 53,951 patients. J Gastroenterol Hepatol 2011;26(4):678-682

9 Zuckerman GR, Trellis DR, Sherman TM, Clouse RE. An objective measure of stool color for differentiating upper from lower gastrointestinal bleeding. Dig Dis Sci 1995;40(8):1614-1621

10 Laine L, Shah A. Randomized trial of urgent vs. elective colonoscopy in patients hospitalized with lower GI bleeding. Am J Gastroenterol 2010;105(12):2636-2641

11 Oakland K, Chadwick G, East JE, et al. Diagnosis and management of acute lower gastrointestinal bleeding: guidelines from the British Society of Gastroenterology. Gut 2019;68(5):776-789

12 Chen WC, Lin KH, Huang YT, et al. The risk of lower gastrointestinal bleeding in low-dose aspirin users. Aliment Pharmacol Ther 2017;45(12):1542-1550 
13 Ali M, Sharma A. Risk of lower gastrointestinal bleeding with low-dose aspirin: to give or not to give? Natl Med J India 2018;31(4):219-221

14 Patel SD, Desai R, Patel U, et al. Thirty-day readmissions after upper and lower gastrointestinal hemorrhage: a national perspective in the United States. J Clin Gastroenterol 2019;53(8):582-590

15 Sheth R, SomeshwarV, Warawdekar G. Treatment of acute lower gastrointestinal hemorrhage by superselective transcatheter embolization. Indian J Gastroenterol 2006;25(6):290-294

16 Aoki T, Yamada A, Nagata N, Niikura R, Hirata Y, Koike K. External validation of the NOBLADS score, a risk scoring system for severe acute lower gastrointestinal bleeding. PLoS One 2018;13(4):e0196514
17 Aoki T, Nagata N, Shimbo T, et al. Development and validation of a risk scoring system for severe acute lower gastrointestinal bleeding. Clin Gastroenterol Hepatol 2016;14(11):1562-1570

18 Anand AC, Patnaik PK, Bhalla VP, Chaudhary R, Saha A, Rana VS. Massive lower intestinal bleeding-a decade of experience. Trop Gastroenterol 2001;22(3):131-134

19 Khurana AK, Saraya A, Jain N, Chandra M, Kulshreshta R. Profile of lower gastrointestinal bleeding in children from a tropical country. Trop Gastroenterol 1998;19(2):70-71

20 Yachha SK, Khanduri A, Sharma BC, Kumar M. Gastrointestinal bleeding in children. J Gastroenterol Hepatol 1996;11(10):903-907 